\title{
Monitoring of HAART regime antiretrovirals in serum of acquired immunodeficiency syndrome patients by micellar liquid chromatography
}

\author{
I. Casas-Breva, ${ }^{a}$ J. Peris-Vicente, ${ }^{* b}$ M. Rambla-Alegre, ${ }^{b}$ S. Carda-Broch ${ }^{b}$ and J. Esteve-Romero ${ }^{b}$
}

Received 2nd March 2012, Accepted 17th July 2012

DOI: $10.1039 / \mathrm{c} 2 \mathrm{an} 35298 \mathrm{~b}$

\begin{abstract}
A methodology based on micellar liquid chromatography to monitor five antiretroviral drugs (lamivudine, stavudine, tenofovir, zidovudine and efavirenz) was proposed. Antiretrovirals were studied in sets of three, corresponding to each highly active antiretroviral therapy (HAART) regime, prescribed to acquired immunodeficiency syndrome (AIDS)-infected patients. Four aqueous micellar mobile phases buffered at $\mathrm{pH} 7$ were optimized to separate these compounds, using sodium dodecyl sulfate as the tensioactive, and 1-propanol or 1-pentanol as the organic modifier. The composition of each mobile phase was optimized for each antiretroviral. The common separation conditions were: $\mathrm{C} 18$ apolar column $(125 \times 4.6 \mathrm{~mm}, 5 \mu \mathrm{m}$ particle size $)$, UV detection set at $214 \mathrm{~nm}$, and mobile phase

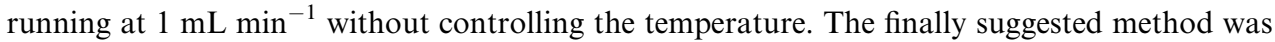
validated for five analysed antiretroviral drugs following the US Food and Drug Administration guidelines in terms of: linearity between 0.5 and $50 \mathrm{ppm}\left(r^{2}>0.9995\right)$, sensitivity (LOD lower than 0.25 $\mathrm{ppm}$ ), intra- and inter-day precision ( $<7.1$ and $<5.2 \%$, respectively) and accuracy (recovery $88.5-$

$105.3 \%$ and $93.5-101.3 \%$, respectively), as well as robustness $(<6.5 \%)$. The proposed method was used to monitor the level of antiretrovirals in the serum of AIDS patients. The suggested methodology was found to be useful in the routine analysis of antiretrovirals in serum samples.
\end{abstract}

\section{Introduction}

Acquired immunodeficiency syndrome (AIDS) is a disease provoked by the human immunodeficiency virus 1 (HIV-1), which strongly affects the human immunological system. ${ }^{1}$ Currently, HIV/AIDS is the fourth greatest cause of death worldwide. It is estimated that 40 million people are infected with HIV and 22 million have died of the disease. ${ }^{2}$ HIV-1 is spread between by blood contact and sexual relationships ${ }^{3}$ and through blood, amniotic fluid and/or breast milk from a mother to her child. ${ }^{4}$ A definitive cure has not been found, but in 1996, the highly active antiretroviral therapy (HAART) was proposed, with impressive clinical results in suppressing the activity of HIV. ${ }^{5}$ HAART involves different kinds of antiretroviral drugs, such as: nucleoside reverse transcriptase inhibitors (NRTIs): abacavir, didanosine, emtricitabine, lamivudine, stavudine, tenofovir, zalcitabine and zidovudine; non-nucleoside reverse transcriptase inhibitors (NNRTIs): delavirdine, nevirapine and efavirenz; and protease inhibitors (PIs): amprenavir, atazanavir, darunavir, fosamprenavir, indinavir, lopinavir, nelfinavir, ritonavir, saquinavir and tipranavir.,

${ }^{a}$ Dep. de Farmàcia Hospitalaria, Hospital la Plana, 12540 Vila-real, Spain ${ }^{b}$ Dep. de Química Física i Analítica, E.S.T.C.E., Universitat Jaume I, Campus Riu Sec, 12071 Castelló, Spain. E-mail: vicentej@qfa.uji.es; Fax: +34 964728066; Tel: +34964728099
HAART regimes contain three or four drugs, two NRTIs and one or two NNRTIs, or one or two PIs. The hydrophobicity is assessed by the quotient of the solubility in octanol and in water $\left(P_{\mathrm{o} / \mathrm{w}}\right)$. A high value of $P_{\mathrm{o} / \mathrm{w}}$ indicates a high hydrophobicity. ${ }^{8}$ The mixtures more administrated are: (a) lamivudine (log $\left.P_{\mathrm{o} / \mathrm{w}}=-1.4\right)$, zidovudine $\left(\log P_{\mathrm{o} / \mathrm{w}}=0.05\right)$ and efavirenz $(\log$ $\left.P_{\mathrm{o} / \mathrm{w}}=4.46\right)$; (b) lamivudine, stavudine $\left(\log P_{\mathrm{o} / \mathrm{w}}=-0.8\right)$ and efavirenz; and (c) lamivudine, tenofovir $\left(\log P_{\mathrm{o} / \mathrm{w}}=-1.6\right)$ and efavirenz. For each patient, one HAART set of antiretrovirals is prescribed. ${ }^{6,9}$

Therapeutic drug monitoring (TDM) consists of the determination of the amount of a drug in the blood. This information can be used by the clinician to establish many aspects of patient treatment, such as malabsorption, drug interactions, individual drug pharmacokinetics and the way each person metabolizes the drug. ${ }^{10}$ This can explain the different effects of the drug depending on the patient and the possible secondary effects. On the other hand, the presence of sub-therapeutic levels of antiretroviral drugs in the blood may provoke the virus to develop resistance. ${ }^{11}$ This can help to determine the proper dose to be taken for a particular person. ${ }^{6}$ However, as each patient takes only one of the HAART regimes, the screening of a wide amount of antiretrovirals is large. Clinics need analytical methods to quantify the three antiretrovirals belonging to the corresponding HAART. The be able to optimize the methodology for a low number of analytes instead of a high number will allow the 
development of a methodology with a lower analysis time and more selectivity, sensitivity and resolution. ${ }^{\mathbf{1 2}}$

We have previously developed a methodology based on micellar liquid chromatography for the screening of 13 antiretroviral drugs by the use of only three mobile phases. ${ }^{6}$ The use of micellar solvents is especially useful in samples with serum matrices, because serum contains hydrophobic compounds which are harmful for the chromatographic system. ${ }^{13-15}$ Micellar media denaturalize and solubilize hydrophobic substances, releasing possible interferent-analyte adducts and avoiding precipitation into the columns. ${ }^{\mathbf{8} 16}$ After a simple dilution and filtration, samples are able to be directly injected, expediting the experimental procedure. ${ }^{17}$ Our previously developed analytical method $^{6}$ can be adapted by the modification of the mobile phases in order to analyze three determined antiretrovirals. This way, this modified methodology will keep the benefits of micellar liquid chromatography, including the use of a low amount of pollutant chemicals. ${ }^{18}$

The aim of this work is to develop an analytical methodology to determine each of the most used sets of three antiretroviral drugs used in HAART in serum. The selected HAART sets of antiretrovirals were: lamivudine + zidovudine + efavirenz, lamivudine + stavudine + efavirenz and lamivudine + tenofovir + efavirenz. The methodology must be validated in terms of linear interval, sensitivity, selectivity, limits of detection and quantification, precision, accuracy and robustness, following the guidelines of the FDA. ${ }^{19}$ The developed analytical method was used to quantify the analytes in human serum from AIDS patients.

\section{Experimental procedure}

\subsection{Chemicals and reagents}

Sodium dodecyl sulphate (SDS, 99\% purity) was obtained from Merck (Darmstadt, Germany). Sodium dihydrogen phosphate monohydrate and $\mathrm{HCl}$ were ordered from Panreac (Barcelona, Spain). $\mathrm{NaOH}$ came from Scharlab (Barcelona, Spain). Methanol was bought from J.T. Baker (Deventeer, The Netherlands) and 1-propanol and 1-pentanol came from Scharlab. An ultrapure water device (Millipore S.A.S., Molsheim, France) was used to prepare the aqueous solutions and mobile phases.

The antiretrovirals studied were: efavirenz (E), lamivudine (L), zidovudine (Z) (purity >99.9\%, Filaxis, Córdoba, Argentina), tenofovir (T), stavudine (S) (purity $>99.9 \%$ Bristol Myers Squibb, New York, NY, USA). The antiretrovirals were studied grouped in sets as follows: mixture $1=\mathrm{L}+\mathrm{Z}+\mathrm{E}$; mixture $2=$ $\mathrm{L}+\mathrm{S}+\mathrm{E}$; and mixture $3=\mathrm{L}+\mathrm{T}+\mathrm{E}$.

\subsection{Equipment and chromatographic conditions}

A Metter-Toledo analytical balance (Greifensee, Switzerland) was used to weigh the analyte. The $\mathrm{pH}$ was measured with a Crison potentiometer (Barcelona, Spain) equipped with a combined $\mathrm{Ag} / \mathrm{AgCl} / \mathrm{glass}$ electrode. An ultrasonic bath was used to dissolve the standards (model Ultrasons-H, Selecta, Abrera, Spain).

Chromatographic separations were performed using an Agilent Technologies Series 1100 system (Palo Alto, CA, USA) equipped with an isocratic pump, a degasser, an autosampler and an absorbance diode array detector (DAD). The stationary phase was in a Kromasil C18 column with the following characteristics: pore size $100 \AA$, length $15 \mathrm{~cm}$, internal diameter $4.6 \mathrm{~mm}$, particle size $5 \mu \mathrm{m}$. Several mobile phases were tested by varying the SDS concentration, the amount of 1-propanol and 1-pentanol, and the $\mathrm{pH}$. The optimal mobile phase composition for each antiretroviral and its corresponding retention time are shown in Table 1. In all cases, the $\mathrm{pH}$ was fixed to 7 using $0.01 \mathrm{M}$ disodium monohydrogen phosphate buffer. Mobile phases run under the isocratic mode at $1 \mathrm{~mL} \mathrm{~min}^{-1}$ without controlling the temperature. The injection volume was $20 \mu \mathrm{L}$ and detection was set at $214 \mathrm{~nm}$. The solutions and the mobile phases were filtered through $0.45 \mu \mathrm{m}$ nylon membranes (Micron Separations, Westboro, MA, USA). Special care was taken for the chromatographic system, due to the use of micellar solutions. ${ }^{8}$

\subsection{Blood collection}

The blood samples used for spiking were taken from healthy, AIDS-infected and heroin addict AIDS-infected volunteers. These samples were collected with DB SST Tubes (BD Vacutainer Systems, Plymouth, UK), centrifuged at $3000 \mathrm{rpm}$ for 10 min and serum was finally separated and either used immediately or frozen and stored at $-20{ }^{\circ} \mathrm{C}$. Blood samples were provided by the La Plana Hospital in Vilarreal and the General Hospital in Castelló, Spain, after consent had been obtained from the Ethical Committee and from patients.

\subsection{Solution and sample preparation}

Mobile phases were prepared by dissolving the appropriate amount of SDS and disodium monohydrogen phosphate in ultrapure water. Then the $\mathrm{pH}$ was adjusted by adding drops of $\mathrm{HCl}$ or $\mathrm{NaOH}$ solutions to reach the desired value. Furthermore, the appropriate volume of organic solvent (1-propanol or 1pentanol) was added and the solution was adjusted to the desired volume with ultrapure water, ultrasonicated and filtered.

Stock solutions of each antiretroviral were prepared in methanol-water 5:95 (v/v). Working solutions were prepared by diluting these stock solutions in ultrapure water. All stock and working solutions stored at $+4{ }^{\circ} \mathrm{C}$ were stable for at least 3 months. ${ }^{6}$

For optimization and validation studies, spiked serum samples were prepared by adding the appropriate volume of stock or working solution to $0.5 \mathrm{~mL}$ of serum and then adjusting the final volume to $5 \mathrm{~mL}$ with a $0.05 \mathrm{M} \mathrm{SDS}$ aqueous solution buffered at $\mathrm{pH}$ 7. The sample was vigorously shaken to favor homogenization and stored for one day in the fridge at $5{ }^{\circ} \mathrm{C}$ to favor the contact between analytes and the sample, and also solvent evaporation. ${ }^{20,21}$ Then the serum was $1 / 10$ diluted.

\subsection{Statistical treatment of the data}

In order to perform the optimization, the selected chromatographic parameters were studied: retention time $\left(t_{\mathrm{R}}\right.$ : time at maximal value of absorbance signal; $\min )$; efficiency $(N$; number of theoretical plates); asymmetry $\left(B / A ; B=\right.$ distance between $t_{\mathrm{R}}$ and the time at $0.1 \times$ signal height at the end of the peak; $A=$ distance between $t_{\mathrm{R}}$ and the time at $0.1 \times$ signal height at the beginning of the peak). These parameters were calculated for each peak from chromatograms using Michrom software. ${ }^{22}$ 
Table 1 Composition of the optimal mobile phase and chromatographic parameters to separate the antiretroviral belonging to each HAART set

\begin{tabular}{|c|c|c|c|c|c|c|}
\hline Mixture & Antiretroviral & Retention time ${ }^{a} / \min$ & SDS amount/M & $\begin{array}{l}\text { Organic modifier } \\
\text { (concentration, \%) }\end{array}$ & $\begin{array}{l}\text { Efficiency } \\
(N)\end{array}$ & $\begin{array}{l}\text { Asymmetry } \\
(B / A)\end{array}$ \\
\hline 1 & $\begin{array}{l}\text { Lamivudine } \\
\text { Zidovudine } \\
\text { Efavirenz }\end{array}$ & $\begin{array}{r}2.05 \pm 0.06 \\
2.58 \pm 0.05 \\
14.20 \pm 0.15\end{array}$ & $\begin{array}{l}0.05 \\
0.05\end{array}$ & $\begin{array}{l}\text { 1-propanol }(2.5 \%) \\
1 \text {-pentanol }(6 \%)\end{array}$ & $\begin{array}{l}2046 \\
2694 \\
2569\end{array}$ & $\begin{array}{l}1.286 \\
1.178 \\
1.062\end{array}$ \\
\hline 2 & $\begin{array}{l}\text { Stavudine } \\
\text { Lamivudine } \\
\text { Efavirenz }\end{array}$ & $\begin{aligned} 2.65 & \pm 0.07 \\
4.96 & \pm 0.09 \\
14.20 & \pm 0.15\end{aligned}$ & $\begin{array}{l}0.05 \\
0.05\end{array}$ & $\begin{array}{l}- \\
1 \text {-pentanol }(6 \%)\end{array}$ & $\begin{array}{l}2462 \\
2172 \\
2569\end{array}$ & $\begin{array}{l}1.202 \\
1.287 \\
1.062\end{array}$ \\
\hline 3 & $\begin{array}{l}\text { Lamivudine } \\
\text { Tenofovir } \\
\text { Efavirenz }\end{array}$ & $\begin{array}{r}2.69 \pm 0.05 \\
4.01 \pm 0.04 \\
14.20 \pm 0.15\end{array}$ & 0.05 & $\begin{array}{l}- \\
1 \text {-pentanol }(6 \%)\end{array}$ & $\begin{array}{l}2009 \\
2060 \\
2569\end{array}$ & $\begin{array}{l}1.103 \\
1.172 \\
1.062\end{array}$ \\
\hline
\end{tabular}

A chromatographic peak is considered satisfactory if the retention time is low, but higher than 2 min (far enough from dead time), the efficiency is high and the asymmetry is close to 1 . Optimization was made by the criterion of obtaining more satisfactory chromatographic peaks (see above) without overlapping. ${ }^{8}$

\section{Results and discussion}

\subsection{Optimization of the separation conditions}

The separation conditions were changed from those proposed in ref. 6 in order to improve the chromatographic parameters (efficiency, asymmetry and retention time) by focusing the study in the three compounds of each HAART mixture potentially
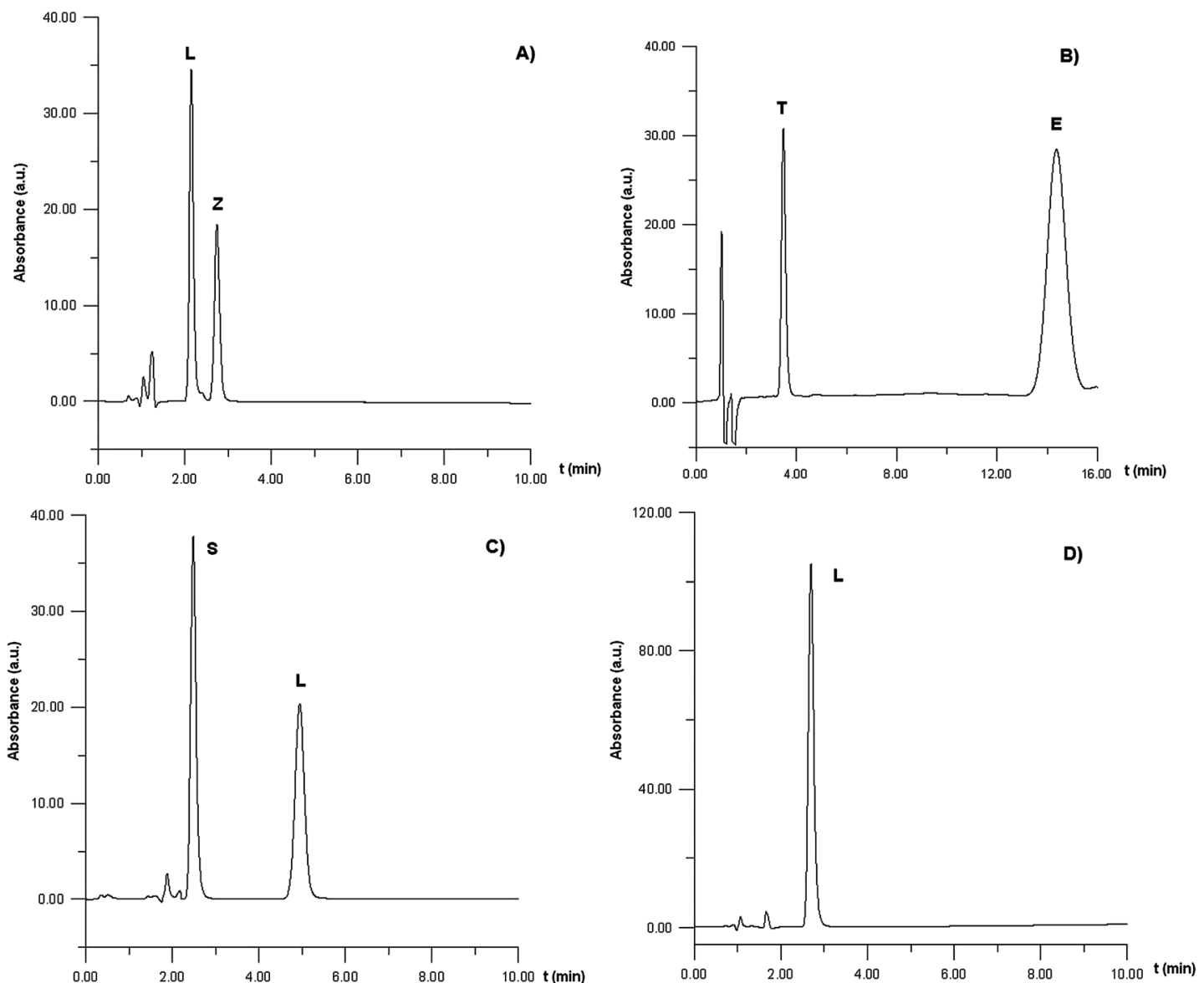

Fig. 1 Chromatograms obtained by analysis of antiretroviral standard (20 ppm) using for each one its optimized mobile phase: (A) 0.05 M SDS/2.5\% 1propanol for lamivudine and zidovudine in mixture 1; (B) $0.05 \mathrm{M} \mathrm{SDS} / 6 \%$ 1-pentanol for tenofovir (in mixture 3) and efavirenz (in all mixtures); (C) 0.05 M SDS for stavudine and lamivudine in mixture 2, and (D) 0.15 M SDS for lamivudine in mixture 3. 
Table 2 Regression curves parameters (slope, intercept and regression coefficient) and sensitivity values (LOD and LOQ) calculated for each studied antiretroviral

\begin{tabular}{llllll}
\hline Mixture & Compound & Slope & Intercept & $r^{2}$ & LOD/ppm \\
\hline 1 & Lamivudine & $37.5 \pm 0.2$ & $5.1 \pm 1.9$ & 0.9999 & 0.18 \\
1 & Zidovudine & $43.5 \pm 0.3$ & $-5 \pm 8$ & 0.9998 & 0.15 \\
$1 / 2 / 3$ & Efavirenz & $58.3 \pm 0.4$ & $-3 \pm 4$ & 0.9997 & 0.10 \\
& & & & & \\
2 & Stavudine & $52.7 \pm 0.3$ & $-5 \pm 9$ & 0.9995 & 0.15 \\
2 & Lamivudine & $35.7 \pm 0.3$ & $4 \pm 2$ & 0.9999 & 0.21 \\
& & & & & \\
3 & Lamivudine & $36.5 \pm 0.2$ & $4 \pm 3$ & 0.9996 & 0.20 \\
3 & Tenofovir & $44.8 \pm 0.3$ & $2 \pm 3$ & 0.9997 & 0.25 \\
\hline
\end{tabular}

administrated to a patient, instead of a wide number of independent antiretrovirals. The study was limited to isocraticrunning mobile phases, because no stabilization time is required between two injections, increasing the robustness and the number of analyzed samples per day. The optimization was performed using standards 1/10-diluted in $0.05 \mathrm{M} \mathrm{SDS}$ at $\mathrm{pH} 7$.

The stationary phase, $\mathrm{pH}$ of the mobile phase and the detection wavelength were set as $\mathrm{C} 18,7$ and $214 \mathrm{~nm}$, respectively, because it was proven in ref. 6 that these conditions provide an adequate separation and sensitivity. Moreover, the selected $\mathrm{pH}$ is inside the working $\mathrm{pH}$ of the column (1.5-9.5). The improvement of the chromatographic parameters was made by varying the mobile phase composition (SDS and organic modifier amount).
Lamivudine, zidovudine, stavudine and tenofovir are quite polar compounds, according to their low $\log P_{\mathrm{o} / \mathrm{w}}$. That means that using a $\mathrm{C} 18$ column and pure mobile phase would provide adequate resolution and retention time. However, a short chain alcohol (as 1-propanol) can be added in order to improve the chromatographic parameters. On the other hand, efavirenz has a high $\log P_{\mathrm{o} / \mathrm{w}}$, indicating that it is more hydrophobic, and would show a high retention time. In this case, an organic modifier (1-butanol or 1-pentanol) should be added to the SDS aqueous mobile phase to obtain useful chromatographic parameters. Then optimization of mobile phases was performed by testing a pure SDS aqueous mobile phase and adding 1-propanol, 1-butanol and 1-pentanol.

In order to find the most adequate mobile phase composition, each antiretroviral (20 ppm standard) was analyzed the following mobile phases, containing SDS $\mathrm{mM} /$ modifier \%: 0.05/0; 0.10/0; 0.15/0; 0.05/1-propanol 2.5; 0.05/1-propanol 12.5; 0.1/1-propanol 7.5; 0.15/1-propanol 2.5; 0.15/1-propanol 12.5; 0.05/1-butanol 1; 0.05/1-butanol 7; 0.1/1-butanol 4; 0.15/1-butanol 1; 0.15/1butanol 7; 0.05/1-pentanol 2; 0.05/1-pentanol 6; 0.1/1-pentanol 4; 0.15/1-pentanol 2 and 0.15/1-pentanol 6 . The obtained chromatographic peaks were used for optimization as indicated in Section 2.5.

The optimized mobile phases are indicated in Table 1, and the chromatograms obtained by the analysis of the standards under the selected chromatographic conditions are shown in Fig. 1.

Table 3 Intra- and inter-day precision and accuracy for the detection of each studied antiretroviral and HAART set obtained by applying the analytical methodology

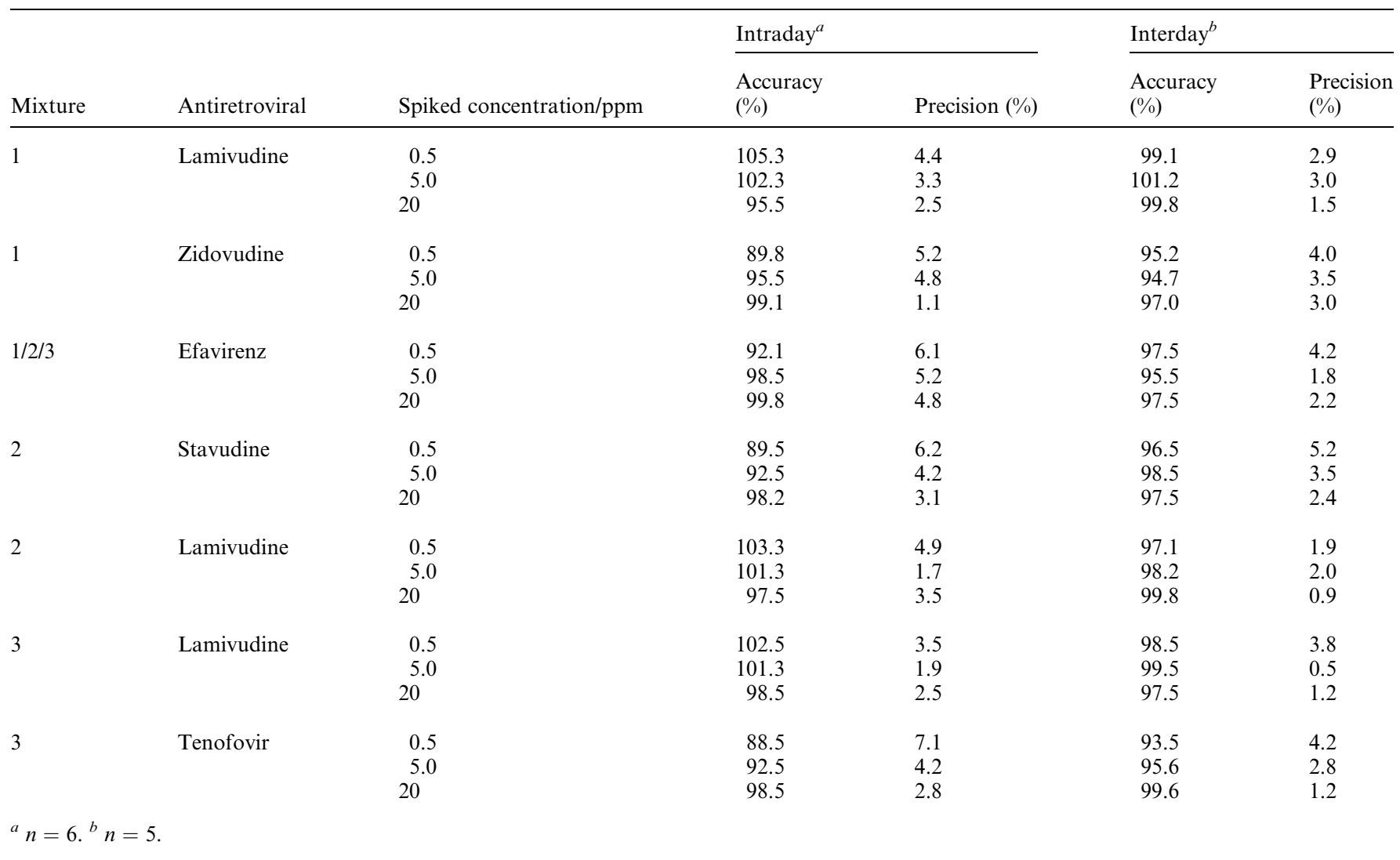


Table 4 Evaluation of the robustness of the micellar liquid chromatography method ${ }^{a}$

\begin{tabular}{|c|c|c|c|c|c|}
\hline Mixture & Antiretroviral & Parameter & Level & Retention time/min (RSD, \%) & Area (arbitrary unit) (RSD, \%) \\
\hline 1 & Lamivudine & $\begin{array}{l}\text { Conc. SDS/mol L } \\
\mathrm{pH} \\
\text { 1-Propanol (\%) } \\
\text { Flow/mL min }\end{array}$ & $\begin{array}{l}0.45-0.55 \\
6.9-7.1 \\
1.9-2.1 \\
0.95-1.05\end{array}$ & $\begin{array}{l}2.11 \pm 0.10(4.7) \\
2.06 \pm 0.09(4.4) \\
2.04 \pm 0.11(5.4) \\
2.05 \pm 0.13(6.3)\end{array}$ & $\begin{array}{l}778 \pm 23(3.0) \\
762 \pm 25(3.3) \\
758 \pm 30(4.0) \\
750 \pm 17(2.3)\end{array}$ \\
\hline 1 & Zidovudine & $\begin{array}{l}\text { Conc. SDS } / \mathrm{mol} \mathrm{L}^{-1} \\
\mathrm{pH} \\
\text { 1-Propanol (\%) } \\
\text { Flow/mL } \mathrm{min}^{-1}\end{array}$ & $\begin{array}{l}0.45-0.55 \\
6.9-7.1 \\
1.9-2.1 \\
0.95-1.05\end{array}$ & $\begin{array}{l}2.60 \pm 0.09(3.5) \\
2.55 \pm 0.11(4.3) \\
2.61 \pm 0.12(4.6) \\
2.52 \pm 0.10(4.0)\end{array}$ & $\begin{array}{l}870 \pm 19(2.2) \\
861 \pm 21(2.4) \\
880 \pm 30(3.4) \\
851 \pm 14(1.6)\end{array}$ \\
\hline $1 / 2 / 3$ & Efavirenz & $\begin{array}{l}\text { Conc. SDS } / \mathrm{mol} \mathrm{L}^{-1} \\
\mathrm{pH} \\
\text { 1-Pentanol } \\
\text { Flow/mL min }\end{array}$ & $\begin{array}{l}0.45-0.55 \\
6.9-7.1 \\
5.9-6.1 \\
0.95-1.05\end{array}$ & $\begin{array}{l}14.4 \pm 0.5(3.5) \\
14.1 \pm 0.4(2.8) \\
14.5 \pm 0.5(3.4) \\
14.2 \pm 0.6(4.2)\end{array}$ & $\begin{array}{l}1200 \pm 50(4.2) \\
1130 \pm 30(2.7) \\
1150 \pm 40(3.5) \\
1190 \pm 30(2.5)\end{array}$ \\
\hline 2 & Lamivudine & $\begin{array}{l}\text { Conc. SDS } / \mathrm{mol} \mathrm{L}^{-1} \\
\mathrm{pH} \\
\text { Flow } / \mathrm{mL} \mathrm{min}^{-1}\end{array}$ & $\begin{array}{l}0.45-0.55 \\
6.9-7.1 \\
0.95-1.05\end{array}$ & $\begin{array}{l}5.01 \pm 0.22(4.4) \\
4.92 \pm 0.15(3.0) \\
4.98 \pm 0.19(3.8)\end{array}$ & $\begin{array}{l}724 \pm 24(3.3) \\
720 \pm 15(2.1) \\
710 \pm 20(2.8)\end{array}$ \\
\hline 3 & Lamivudine & $\begin{array}{l}\text { Conc. SDS } / \mathrm{mol} \mathrm{L}^{-1} \\
\mathrm{pH} \\
\text { Flow/mL min }\end{array}$ & $\begin{array}{l}0.145-0.155 \\
6.9-7.1 \\
0.95-1.05\end{array}$ & $\begin{array}{l}2.72 \pm 0.11(4.0) \\
2.67 \pm 0.08(3.0) \\
2.70 \pm 0.09(3.3)\end{array}$ & $\begin{array}{l}750 \pm 24(3.2) \\
731 \pm 19(2.6) \\
742 \pm 16(2.2)\end{array}$ \\
\hline 3 & Tenofovir & $\begin{array}{l}\text { Conc. SDS } / \mathrm{mol} \mathrm{L}^{-1} \\
\mathrm{pH} \\
\text { 1-Pentanol } \\
\text { Flow/mL } \mathrm{min}^{-1}\end{array}$ & $\begin{array}{l}0.45-0.55 \\
6.9-7.1 \\
5.9-6.1 \\
0.95-1.05\end{array}$ & $\begin{array}{l}4.06 \pm 0.13(3.2) \\
4.04 \pm 0.09(2.2) \\
3.95 \pm 0.13(3.0) \\
4.00 \pm 0.17(4.3)\end{array}$ & $\begin{array}{l}870 \pm 30(3.4) \\
910 \pm 19(2.1) \\
890 \pm 24(2.7) \\
900 \pm 15(1.7)\end{array}$ \\
\hline${ }^{a} n=6$. & & & & & \\
\hline
\end{tabular}

3.1.1 Separation conditions for lamivudine and efavirenz. As these two compounds are in the three mixtures, and their results are firstly discussed.

Lamivudine shows a low retention time in all cases (according to its polarity). Mobile phases providing a retention time lower than 2.00 min were not considered. In this case, it elutes too close to the dead time, and the probability of overlapping with matrix compounds is high. Only a pure aqueous mobile phase, $0.05 \mathrm{M}$ SDS/1-propanol 2.5\% and $0.05 \mathrm{M} \mathrm{SDS} / 1$-butanol $1 \%$ were able to elute lamivudine with a retention time of more than $2 \mathrm{~min}$.

Efavirenz provides a high retention time in all cases (in agreement with its low polarity). Mobile phases eluting the analyte with a retention time of more than $20 \mathrm{~min}$ were not considered. Indeed, a greater retention time would lower the
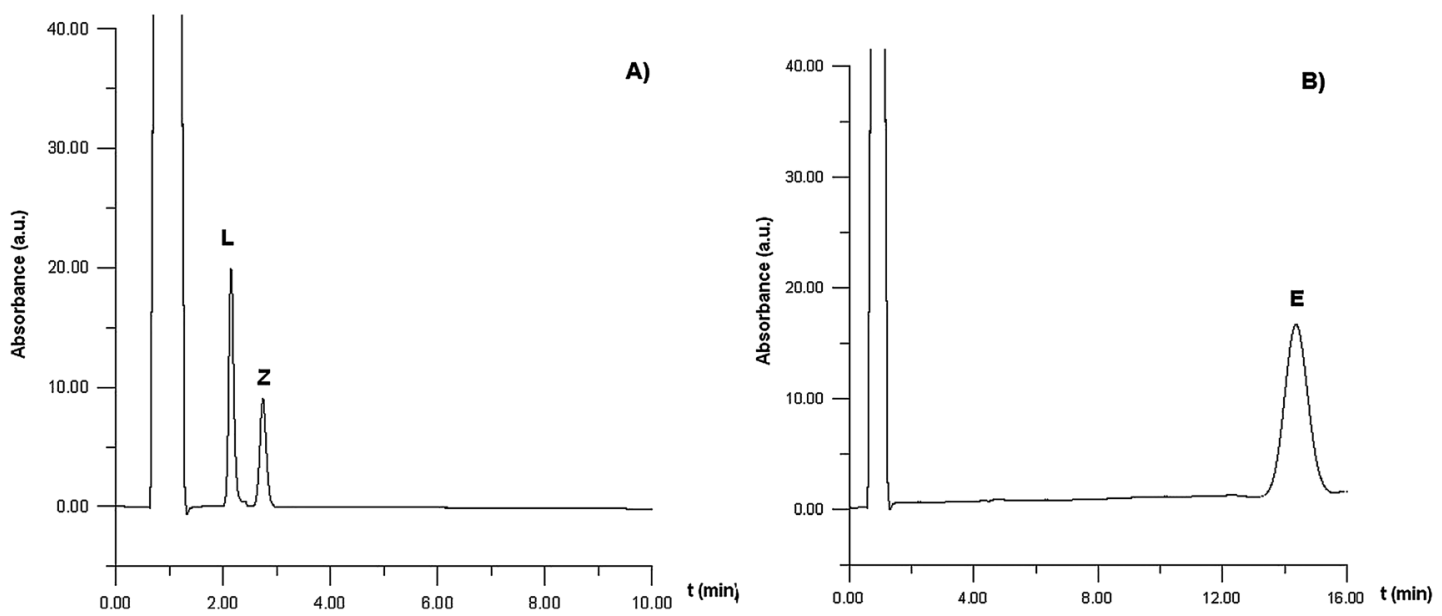

Fig. 2 Chromatograms obtained by the analysis of a serum sample of the AIDS patient no. 505459, who takes the HAART regime composed of lamivudine, zidovudine and efavirenz (see Table 5 for quantitative values). 
Table 5 Amount of antiretroviral found (ppm) in AIDS patient serum samples $^{a}$

\begin{tabular}{lccc}
\hline \multicolumn{4}{l}{ HAART regime prescribed $=$ mixture 1} \\
\hline Patient & Lamivudine & Zidovudine & Efavirenz \\
\hline 576704 & $5.6 \pm 0.4$ & $27.7 \pm 1.1$ & $125 \pm 8$ \\
505933 & $13.7 \pm 0.9$ & $36 \pm 3$ & $23 \pm 3$ \\
519824 & $179.5 \pm 1.5$ & $12.8 \pm 1.8$ & Under LOD \\
521320 & $5.3 \pm 1.5$ & $32.8 \pm 0.8$ & $393 \pm 9$ \\
520287 & $12 \pm 4$ & $29.0 \pm 0.3$ & $84 \pm 6$ \\
516064 & $7.0 \pm 0.9$ & $45.4 \pm 1.8$ & Under LOD \\
505459 & $104 \pm 8$ & $89 \pm 5$ & $122 \pm 7$ \\
\hline
\end{tabular}

HAART regime prescribed $=$ mixture 2

\begin{tabular}{llrr}
\hline Patient & Lamivudine & Stavudine & Efavirenz \\
\hline 521787 & $35 \pm 4$ & $130 \pm 15$ & $510 \pm 40$ \\
521321 & $31 \pm 5$ & $72 \pm 9$ & $183 \pm 22$
\end{tabular}

HAART regime prescribed $=$ mixture 3

\begin{tabular}{lclr} 
Patient & Lamivudine & Tenofovir & Efavirenz \\
\hline 532374 & $16.6 \pm 1.6$ & Under LOD & $91 \pm 6$ \\
532378 & $34.3 \pm 1.6$ & Under LOD & $305 \pm 13$ \\
579254 & $26.7 \pm 0.6$ & Under LOD & $1010 \pm 50$ \\
579253 & $14.2 \pm 0.6$ & Under LOD & $660 \pm 40$ \\
519821 & $34.9 \pm 1.0$ & Under LOD & $38 \pm 4$ \\
516060 & $20.6 \pm 2.1$ & Under LOD & $365 \pm 21$ \\
505948 & $49 \pm 3$ & Under LOD & $150 \pm 12$ \\
514486 & $30.7 \pm 1.2$ & Under LOD & $24 \pm 3$ \\
505050 & $29.3 \pm 2.0$ & Under LOD & $13.1 \pm 0.4$ \\
505045 & $41.3 \pm 1.4$ & Under LOD & $160 \pm 7$ \\
520289 & $24 \pm 3$ & Under LOD & $159 \pm 9$ \\
${ }^{a} n=5$. & & & \\
& & &
\end{tabular}

efficiency and is not suitable for routine analysis, where a high amount of samples should usually be analyzed. Following this criterion, only $0.15 \mathrm{M} / 1$-propanol $12.5 \%, 0.15 \mathrm{M} / 1$-butanol $7 \%$, $0.05 \mathrm{M}$ SDS/1-pentanol 6\%, 0.1 M SDS/1-pentanol 4\% and $0.15 \mathrm{M} \mathrm{SDS} / 1$-pentanol $6 \%$ were considered.

As seen, the resolution of a mixture of lamivudine and efavirenz is not possible in a pure mobile phase, because of the strong difference of hydrophobicities. Therefore, for each mixture two mobile phases would be needed. The final selection of the optimal mobile phase was made separately for each HAART mixture, depending on the chromatographic parameter of the third compound (zidovudine, stavudine and tenofovir). The chromatographic parameters for each analyte in its optimized mobile phase are shown in Table 1.

3.1.2 Mixture 1: lamivudine + zidovudine + efavirenz. In all tested mobile phases, lamivudine and zidovudine are eluted at similar retention times. In fact, they overlap in all mobile phases except in 0.15 M SDS and 0.05 M SDS/1-propanol 2.5\% where an adequate separation is observed. Finally, 0.05 M SDS/1propanol $2.5 \%$ was selected as it provides a lower retention time and maximum separation between the analytes.

Efavirenz was analyzed using other mobile phase. Among those indicated in Section 3.1.1, 0.05 M SDS/1-pentanol 6\% was considered as optimal because it shows the maximal efficiency $(N=2569)$ and asymmetry $(B / A=1.062)$.
3.1.3 Mixture 2: lamivudine + stavudine + efavirenz. Lamivudine and stavudine show a similar behaviour in the studied mobile phases; in fact, they are resolved only in aqueous mobile phases and they co-elute when an organic modifier is added. The mobile phase $0.05 \mathrm{M}$ SDS was considered as optimal as it provides the maximal separation between the two analytes, maintaining a low retention time (less than $5 \mathrm{~min}$ ). For efavirenz, the same mobile phase as selected in Section 3.1.1 was taken.

3.1.4 Mixture 3: lamivudine + tenofovir + efavirenz. Tenofovir cannot be eluted using aqueous mobile phases, as it shows too high a retention time. Lamivudine and tenofovir can be analyzed together only in $0.05 \mathrm{M} / 1$-propanol $2.5 \%$. However, the retention time was found to be high $(\approx 12.46 \mathrm{~min})$ and the efficiency was $N=1889$. Finally, tenofovir was analyzed using the optimal mobile phase deduced in Sections 3.1.1 and 3.1.2 for efavirenz (0.05 M SDS/1-pentanol 6\%), were it provides a more useful retention time $(\approx 4.01 \mathrm{~min})$ and higher efficiency $(N=$ 2060). Then this mobile phase was taken as optimal for the quantification of tenofovir and efavirenz.

In this case, lamivudine was identified under other chromatographic conditions. The selected mobile phase was aqueous 0.15 M SDS without organic modifier, providing the elution of the analyte at a low time $(\approx 2.69 \mathrm{~min})$ but with enough separation from the dead time and with adequate efficiency $(N=2009)$.

3.1.5 Separation of standard and matrix compounds. Blank serum samples (free of antiretrovirals) were studied in order to assess if the analytes overlap with other compounds of the matrix. In order to ensure that the tested blank serum samples do not contain any antiretroviral, these samples were taken from ten healthy volunteers (five males and five females) who have never taken any of the studied drugs. Results (not shown) were found to be similar for all the blanks. Despite the complexity of the serum matrices, no peaks were detected with a retention time of $>2.00 \mathrm{~min}$, but the front of the chromatograms showed a very high absorbance. This can be explained by the use of micellar media. Serum hydrophobic compounds are solubilized by their introduction into the micelles (hydrophobic environment). When the blank serum/micellar dilution sample is injected into the chromatographic system, these interactions remain in the micellar mobile phase, strongly decreasing the interaction between these compounds and the stationary phase, and then shortening the elution time. These results have already been found in previous studies on the analysis of serum samples using micellar mobile phases. ${ }^{13-15}$ Therefore, there are no compounds that could overlap with the studied antiretrovirals because their retention times were higher than $2.00 \mathrm{~min}$, far enough from the front of the chromatogram. Then in the spiked samples, the chromatographic peaks corresponding to the antiretrovirals may be observed as sufficiently separated between them and the other peaks, thus avoiding overlapping. This proves the selectivity of the method.

\subsection{Method validation}

Validation was carried out following the guidelines of the FDA. ${ }^{19}$ The evaluated parameters were: linearity, limits of detection (LOD) and quantification (LOQ), precision, accuracy and 
robustness. As lamivudine is resolved using three different separation conditions depending on the HAART mixture, and its validation was performed for each mobile phase. The whole calibration was performed using a spiked sample serum (initially free of antiretroviral) from a healthy volunteer. In all cases, the preparation of the spiked serum samples has been performed as explained in Section 2.4.

3.2.1 Linearity and sensitivity. For calibration purposes, a blank serum sample was spiked at nine concentration levels between 0.5 and $50 \mathrm{ppm}$. The calibration was performed separately for each antiretroviral.

The slope, intercept and regression level $\left(r^{2}\right)$ of the calibration curves were obtained by linear correlation between the areas of the chromatographic peaks of each analyte $v$. the concentration by unweighted least-square linear regression. Each calibration level was repeated six times. Calibration was repeated five times (preparing the samples on each occasion) on different days over a period of two months. The regression curve of each analyte, taken as the average of the five measurements is shown in Table 2. The considered absorbance was in arbitrary units and concentration was in ppm.

The LOD is defined as the lowest concentration in the serum which provides a significant signal above the noise of the signal. It is taken as 3 times the standard deviation of the blank (LOD, $3 s$ criterion). The LOQ is defined as the lower amount of antiretroviral which can be reliably quantified and is set as the lower concentration reached in the calibration curve. ${ }^{19}$ The LOD value calculated for each analyte is shown in Table 2. The LOQ was $0.5 \mathrm{ppm}$ for the studied antiretroviral.

3.2.3 Precision and accuracy. The intra- and inter-day precision and accuracy of the proposed methodology were calculated for each antiretroviral and HAART set. These parameters were determined at $0.5,5$ and $20 \mathrm{ppm}$ using spiked serum samples.

The precision was determined as the standard deviation of the provided signal (RSD, \%), whereas the accuracy was calculated as the recovery, i.e. the ratio between the detected and added concentration. The intra-day analysis was determined by injecting aliquots of these samples six times on the same day, while the inter-day analyses correspond to the average of five measurements of the intra-day values taken over a two-month period. The results are shown in Table 3. For the quantification of the five studied antiretrovirals, intra- and inter-day precision were $<7.1 \%$ and $<5.2 \%$, respectively, whereas intra- and inter-day accuracy were between 88.5 and $105.3 \%$ and between 93.5 and $101.3 \%$, respectively. These values are within the limits proposed by the FDA guidelines, which accepts a maximal RSD of $15 \%$ for precision and a recovery of between 80 and $120 \%{ }^{19}$

3.2.4 Robustness. The robustness of the method was performed by analyzing serum samples $(n=3)$ spiked with $20 \mathrm{ppm}$ of each studied antiretroviral, by making slight changes to the following chromatographic conditions (with the others remaining constant each time): SDS amount, quantity of organic modifier, $\mathrm{pH}$ and flow rate. The variation of retention time and peak area were studied. The robustness was examined for each antiretroviral and each optimized mobile phase. Insignificant differences in peak areas and less variability in retention time were observed (see Table 4). Results indicate that the selected factors remain unaffected by small variations in these parameters; the RSD was below $6.5 \%$.

\subsection{Analysis of real samples}

The proposed method was applied to determine the levels of antiretrovirals in serum samples from several AIDS patients from a local Hospital. Only one kind of HAART regime has been prescribed to each patient. For confidentiality reasons, no more information about this can be provided. Results have been organized depending on the set of antiretrovirals they take, as can be seen in Table 5. Results have been shown considering the 1/10-dilution performed in the experimental procedure (Section 2.4). In the case of the measured amount of an antiretroviral upper to $50 \mathrm{ppm}$, an extra 1/10-dilution was carried out. Fig. 2 shows the chromatogram of a patient to whom the mixture 1 HAART (lamivudine, zidovudine and efavirenz) has been prescribed. Antiretrovirals were detected and quantified without interferences. According to the results, the patient effectively takes the medication.

\section{Conclusions}

Micellar liquid chromatography has been proven as a valuable technique to monitor sets of antiretroviral drugs belonging to the three HAART regimes mainly prescribed by doctors for AIDS patients. Analysis was performed without long and tedious extractions and/or sample cleaning, by the direct injection of the sample after a simple dilution and filtration of the matrix. The suggested methodology allows the separation of the analytes in less than $15 \mathrm{~min}$, with adequate efficiency and asymmetry. Moreover, validation was performed according to FDA guidelines with satisfactory results in selectivity, linearity, sensitivity, precision, accuracy and robustness. As low amount of organic solvent and biodegradable salts were used, and the method can be considered as environmentally friendly. Besides, the instrumentation is relatively inexpensive and a high amount of samples can be successively analyzed using an autosampler, making it more attractive in routine analysis for a hospital laboratory.

\section{Acknowledgements}

This work was supported by the Fundació Caixa Castelló-Bancaixa P1-1B2006-12 projects and MEC CTQ 200764473/BQU. M.R.-A. also wishes to thank the MEC for her FPU grant.

\section{References}

1 M. Popovic, M. G. Sarngandharan, E. Read and R. C. Gallo, Science, 1984, 224, 497-500.

2 E. A. Pereira, G. A. Micke and M. F. M. Tavares, J. Chromatogr., A, 2005, 1091, 169-179.

3 D. J. Volsky, K. Sakai, M. Stevenson and S. Dewhurst, AIDS Res., 1986, 2, S35-S48.

4 M. Xu, C. A. White and M. G. Bartlett, J. Liq. Chromatogr. Relat. Technol., 2008, 31, 482-496.

5 S. Broker, Antiviral Res., 2010, 85, 1-18.

6 M. A. Raviolo, I. Casas-Breva and J. Esteve-Romero, J. Chromatogr., $A, 2009$, 1216, 3546-3552.

7 D. R. Weller, R. C. Brundage, H. H. Balfour and H. E. Vezina, J. Chromatogr., B: Anal. Technol. Biomed. Life Sci., 2007, 848, 369-373. 
8 M. Rambla-Alegre, J. Peris-Vicente, S. Marco-Peiró, B. BeltránMartinavarro and J. Esteve-Romero, Talanta, 2010, 81, 894-900.

$9 \mathrm{http} / / /$ www.drugbank.ca/drugs/DB00709 (last accessed 25/02/2012).

10 N. L. Rezk, R. R. Tidwell and A. D. M. Kashuba, J. Chromatogr., B. Anal. Technol. Biomed. Life Sci., 2003, 791, 137-147.

11 P. Garcia de Olalla, H. Knobel, A. Carmona, A. Guelar, J. L. LopezColomes and J. A. Cayla, JAIDS, J. Acquired Immune Defic. Syndr., 2002, 30, 105-110.

12 M. Rambla-Alegre, S. Marco-Peiró, J. Peris-Vicente, B. BeltránMartinavarro, M. A. Collado-Sánchez, S. Carda-Broch and J. Esteve-Romero, Food Chem., 2011, 129, 614-618.

13 J. Esteve-Romero, E. Ochoa-Aranda, D. Bose, M. Rambla-Alegre, J. Peris-Vicente and A. Martinavarro-Domínguez, Anal. Bioanal. Chem., 2011, 397, 1557-1561.

14 E. O. Aranda, J. Esteve-Romero, M. Rambla-Alegre, J. Peris-Vicente and D. Bose, Talanta, 2011, 84, 314-318.

15 S. Marco-Peiró, B. Beltrán-Martinavarro, M. Rambla-Alegre, J. Peris-Vicente and J. Esteve-Romero, Talanta, 2012, 88, 617-622.
16 B. Beltrán-Martinavarro, J. Peris-Vicente, S. Marco-Peiró, J. EsteveRomero, M. Rambla-Alegre and S. Carda-Broch, Analyst, 2012, 137, 269-274.

17 M. Rambla-Alegre, J. Peris-Vicente, J. Esteve-Romero and S. CardaBroch, Food Chem., 2010, 123, 1294-1305.

18 M. L. Chin-Chen, D. Bose, J. Esteve-Romero, J. Peris-Vicente, M. Rambla-Alegre and S. Carda-Broch, Open Anal. Chem. J., 2011, 5, 22-26.

19 Guidance for Industry, Bioanalytical Method Validation. U.S. Department of Health and Human Services. Food and Drug Administration, Rockville, MD, USA, May 2001.

20 G. Cano-Sancho, S. Marin, A. J. Ramos, J. Peris-Vicente and V. Sanchis, Rev. Iberoam. Micol., 2010, 27, 130-135.

21 J. Peris Vicente, J. V. Gimeno Adelantado, M. T. Doménech Carbó, R. Mateo Castro and F. Bosch Reig, Talanta, 2004, 64, 326-333.

22 J. R. Torres-Lapasió, Michrom Software, Marcel-Dekker, New York, USA, 2000. 